\title{
AN INVAR-BASED FABRY-PEROT CAVITY REFRACTOMETER WITH A GALLIUM FIXED-POINT CELL FOR ASSESSMENT OF PRESSURE
}

\author{
I. Silander ${ }^{1}$, C. Forssén ${ }^{1,2}$, J. Zakrisson ${ }^{1}$, M. Zelan ${ }^{2}$, O. Axner ${ }^{1}$ \\ ${ }^{1}$ Department of Physics, Umeå University, SE-901 87 Umeå, Sweden, \\ isak.silaner@umu.se, clayton.forssen@umu.se, johan.zakrisson@umu.se, ove.axner@umu.se \\ ${ }^{2}$ Measurement Technology, RISE Research Institutes of Sweden, SE-501 15 Borås, Sweden, martin.zelan@ rise.se
}

\begin{abstract}
:
An Invar-based Fabry-Perot cavity refractometer equipped with an automated, miniaturized gallium fixed-point cell for assessment of pressure is presented. The use of an Invar cavity spacer has previously demonstrated pressure assessments with sub-0.1 ppm precision. The fixed-point cell, whose design and implementation are presented here, provides a reference for temperature assessment of the gas inside the cavity with an uncertainty of $4 \mathrm{ppm}$. This opens up for a self-contained system for realization of the Pascal with an accuracy in the low ppm range. This is an important step towards disseminating the Pascal through fundamental principles.
\end{abstract}

Keywords: Refractometry, Fabry-Perot cavity, Temperature, Gallium.

\section{INTRODUCTION}

With the recent revision of the SI-system, alternative paths to realize Pascal have become feasible. By measuring the refractivity and the temperature of a gas one can calculate its pressure by the use of the Lorentz-Lorenz equation and an equation of state [1]. Such an instrument would not rely on any mechanical actuator; instead, if wellconstructed, its performance could depend solely on how well the molar refractivity and the gas temperature can be assessed [2].

We have previously developed an innovative method for refractometry, termed gas-modulation refractometry (GAMOR), that allows for a significant reduction of the influence of drifts and fluctuations of the system [3-5]. This has opened up for the use of non-conventional cavity-spacer materials that otherwise were considered unsuitable due to a high thermal expansion. A system realized around an Invar-based dual Fabry-Perot cavity (DFPC) refractometer has been constructed and presented [6].

As discussed in detail in that work, in comparison to cavities based on conventional materials [Zerodur ${ }^{\circledR}$ and ultra-low expansion glass (ULE®)], such a system has several advantages, mainly:
(1) a high ratio of thermal conductivity and volumetric heat capacity, which implies that the spacer will have smaller thermal gradients;

(2) a high volumetric heat capacity, which reduces temperature fluctuations;

(3) a Young's modulus that gives a smaller pressureinduced deformation;

(4) potentially a lower degree of He diffusivity and permeation; and

(5) it can be machined in a standard metal workshop, whereby more complicated geometries can be created more swiftly to a low cost.

For an empty cavity, the system could demonstrate an Allan deviation of $0.03 \mathrm{mPa}$. When assessing pressure at $4303 \mathrm{~Pa}$, the system provided a minimum Allan deviation of $0.34 \mathrm{mPa}$, which corresponds to a relative deviation (or a precision) of $0.08 \mathrm{ppm}$. However, the system, as realized in Ref. [6], assessed temperature by the use of uncalibrated Pt-100 sensors, which gave it a limited accuracy for pressure assessments.

For a wider dissemination of the method, the temperature needs to be assessed with high accuracy and be traceable to a thermometric standard, preferably without any calibration chain. To realize such an instrumentation, we present here an Invarbased DFPC refractometer that has been equipped with an automated temperature regulating and assessment system. Similar to what previously has been demonstrated by deployment on the International Space Station (ISS) [7], the latter employs an automated, miniaturized, custom-made fixed-point cell based upon the well-defined melting point of gallium. By the use of a thermocouple working close to zero temperature difference, the temperature of the cavity assembly is constantly assessed with respect to this melting point.

The use of an Invar-based DFPC facilitates, according to the first two points of above, i.e. (1) and (2), the temperature regulation of the cavity. Referencing the temperature to a gallium fixed-point cell improves on the accuracy of the system. The combination of an Invar-spacer, the GAMOR methodology, 
and a Ga fixed-point cell therefore provides a basis for a self-contained refractometer that in the future only needs a pure gas supply and a frequency reference to realize the Pascal.

\section{THEORY}

It has previously been shown that for a DFPC refractometer the refractivity can be expressed as $[4,6]$

$$
n-1=\frac{\overline{\Delta f}+\overline{\Delta q_{1}}}{1-\overline{\Delta f}+\varepsilon},
$$

where $\overline{\Delta f}$ is the relative shift of the beat frequency given by $\Delta f / v_{01}$, where, in turn, $\Delta f$ is the shift of the beat frequency and $v_{01}$ is the empty cavity frequency of the measurement laser, and $\overline{\Delta q_{1}}$ represents $\Delta q_{1} / q_{01}$, where $\Delta q_{1}$ is the change in mode number, $q_{01}$, of the mode addressed in the empty measurement cavity when gas is let into the cavity. $\varepsilon$ is a normalized cavity deformation, defined as $\left(\delta L / L_{0}\right) /(n-1)$, where $\delta L$ and $L_{0}$ are the pressure induced length deformation and the empty cavity length, respectively.

For pressure up to atmospheric pressure, the gas density $\rho$ can be calculated from the refractivity by use of the extended Lorentz-Lorenz equation,

$$
\rho=\frac{2}{2 A_{R}}(n-1)\left[1+b_{n-1}(n-1)\right],
$$

where $A_{R}$ and $b_{n-1}$ are the molar dynamic polarizability and a series expansion coefficient, respectively. The latter is given by $-\left(1+4 B_{R} / A_{R}^{2}\right) / 6$, where, in turn, $B_{R}$ is the second refractivity virial coefficient in the Lorentz-Lorenz equation.

Up to atmospheric pressure, the pressure can be obtained from the density and temperature as

$$
P=R T \rho\left[1+B_{\rho}(T) \rho\right],
$$

where $R$ is the ideal gas constant, $T$ is the temperature of the gas, and $B_{\rho}(T)$ is the second density virial coefficient.

If higher pressures are to be assessed, additional terms need to be added in the Eqs. (2) and (3).

\section{EXPERIMENTAL SETUP}

\subsection{The Invar-based DFPC-refractometer}

The Invar-based DFPC-refractometer used in this work is, to a large extent, identical to the one presented in detail in a recent publication [6]. The heart of the system is the cavity spacer, which is machined from a rod of Invar (see Fig. 1). In the spacer, two holes for the measurement and reference cavities, and three holes for temperature probes (seen on the short end and on the top of the cavity assembly, respectively), have been drilled. To each of the cavities, a vacuum tube is connected in order to either supply or evacuate the cavity. To mount the cavity mirrors to the spacer the mirrors were placed in a machined inset with an O-ring above. To provide vacuum tight seals, plates were screwed into the spacer to press the mirrors, via the O-rings, onto the spacer.

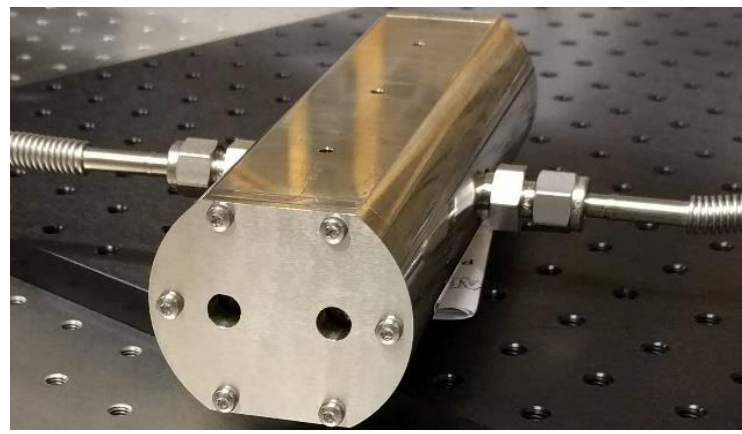

Figure 1: The naked Invar cavity assembly before being equipped with temperature probes and mounted inside the aluminium enclosure (the oven).

The cavity spacer is placed in an aluminium enclosure (referred to as an oven) and supported by four sharp pegs at the points of minimum deflection. On top of the enclosure, four pneumatic vacuum valves, used to control the flow of gas via vacuum tubing to and from the cavities, are placed. A picture of the enclosure is shown in Fig. 2. The enclosure does not only support the valves and fixates the cavity; it also plays a crucial role in the temperature measurement and stabilization system as a regulative oven.

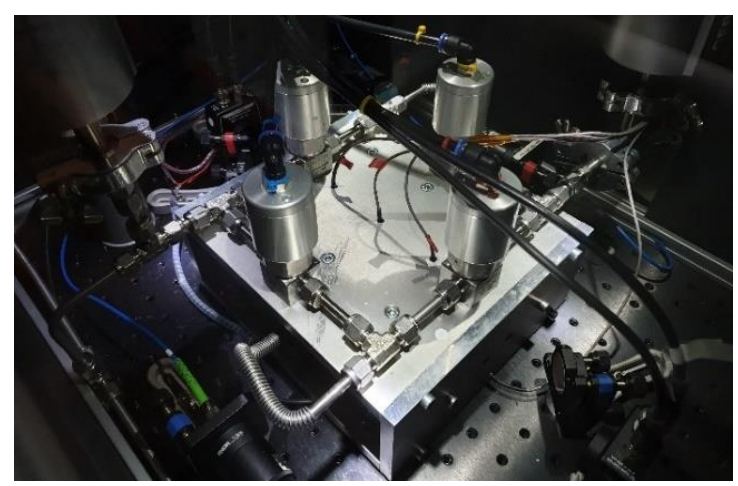

Figure 2: The metal enclosure (the oven), containing the Invar cavity. The valve assembly is attached to the temperature-controlled enclosure to reduce thermal gradients from the gas lines attached to the cavity.

\subsection{Temperature stabilization and assessment}

The temperature stabilization and assessment system, schematically illustrated in Fig. 3, stabilizes the cavity "close" to the temperature corresponding to the melting-point of gallium $\left(T_{G a}=302.9146 \mathrm{~K}\right)$, and then independently monitors the actual cavity temperature, which is considered an adequate representative of the gas temperature, $T$, by measuring the difference in temperature between a fixed-point cell and the cavity by the use of a type-T thermocouple. 




Figure 3: Schematic illustration of the thermal management system. The temperatures of the cavity assembly (left) and the gallium fixed-point cell (right) are controlled by separate PID controllers.

The temperature stabilization is performed in three stages. In the first, the measurement environment is heated to a temperature close to $T_{G a}$ by heating the breadboard on which the enclosure rests by a heat mat. In the second stage, the temperature of the oven is measured with a Pt-100 probe inserted in the bottom of the enclosure. This temperature reading is used to apply feedback to four Peltier elements, placed under the oven, that regulate the oven temperature. In the third stage, the temperature of the cavity spacer, measured as the mean of three Pt-100 probes that are inserted into the cavity spacer, $T_{c a v}$, is used to apply feedback to the set point of the oven servo stage. This feedback aims at keeping the spacer in thermal equilibration with the oven at (or close to) the Ga melting point.

The fixed-point cell consists of a Ga sample (7N Aldrich MKCH6524) housed in a $15 \mathrm{ml}$ Falcon tube. The tube is mounted in a heating block that, in turn, is mounted on top of a Peltier element. A probe assembly, which consists of a $3 \mathrm{~mm}$ folded Kynar ${ }^{\circledR}$ (polyvinylidene fluoride, PVDF) tube heat shrunk around a Pt-100 probe, is used to monitor the temperature of the gallium cell, $T_{\text {cell }}$. The measurement junction of the thermocouple is immersed into the gallium.

The temperature of the heating block, $T_{\text {block }}$, is regulated to a temperature slightly above the meltingpoint of gallium, (ca. $50 \mathrm{mK})$ by applying feedback to the Peltier element. Whenever the temperature of the cell rises $15 \mathrm{mK}$ above $T_{G a}$, the $\mathrm{Ga}$ is considered melted and the cell is automatically reset by a refreezing procedure. In this, the temperature of the block is set to $25{ }^{\circ} \mathrm{C}$. When the cell temperature reaches $25.1{ }^{\circ} \mathrm{C}$, at which the gallium is considered to be frozen, the temperature of the block is again increased to a temperature slightly above $T_{G a}$.

The length of the melting cycle of the Ga depends on the temperature at which the heat block is held, but lasts typically 80 hours for a temperature of the heat block that is $50 \mathrm{mK}$ above the Ga melting point. The time for refreezing is typically less than 1 hour.

The thermocouple, whose voltage is assessed by a nanovoltmeter (Keithley, 2182A), is used to assess the temperature difference between the cavity and the gallium cell, $\Delta T_{T C}$. When on the Ga melting plateau, the temperature of the cavity measured by the thermocouple, $T_{T C}$, is given by $\Delta T_{T C}+T_{G a}$. To reduce temperature overhearing, its reference junctions at the cavity are wound around the spacer a multitude of turns.

\section{RESULTS}

\subsection{Allan deviation of pressure assessments performed by the Invar-based refractometer}

Figure 4 provides a comparison between the Invar-based system and a similar system based on a Zerodur cavity [4]. Before the Ga fixed-point cell was implemented, for each cavity system, two measurement series were taken at dissimilar pressures; one when the system was evacuated, indicated in the form of Allan deviations in Fig. 4 by the magenta and yellow markers, and one at $4303 \mathrm{~Pa}$ provided by a pressure balance, represented by the blue and red markers [6]. The data from the Invar and Zerodur cavities are presented by magenta and blue, and, yellow and red markers, respectively.

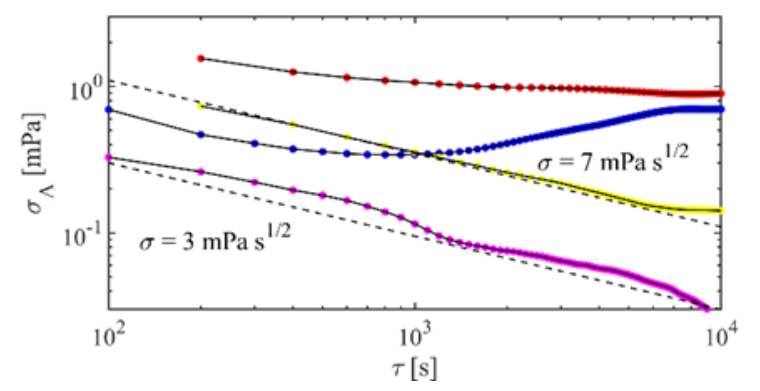

Figure 4: Allan deviations of pressure assessments made by a previous Zerodur-based system (red upper markers) and the Invar-based system (blue), both at $4303 \mathrm{~Pa}$. The yellow and purple (lower) markers illustrate the corresponding empty cavity measurements. Reproduced with modifications with permission from [6].

Compared to the Zerodur-based DFPC, the Invarcavity based refractometer demonstrated an improved stability at both pressures, on all time scales.

For the empty cavity assessments, the implementation of an Invar-based DFPC provided a significantly improved white-noise behaviour. As is discussed in more detail in Ref. [6], this is attributed to higher mechanical stability of the system.

For the pressure assessment at $4303 \mathrm{~Pa}$, and for integration times between 400 and $1100 \mathrm{~s}$, a performance with an Allan deviation below $0.43 \mathrm{mPa}$ (representing sub-0.1 ppm levels) could be demonstrated, attributed to the improved thermal 
conditions of the Invar material. For details about these responses, see Ref. [6] in which a more rigorous scrutiny is presented.

It should be noted though that, since the Pt-100 sensors used at the time when the data shown in Fig. 4 were taken had not been calibrated, the accuracy of the temperature assessment of the gas (and thereby the pressure) was, at that time, estimated to solely be slightly below 400 ppm [6].

\subsection{Evaluation of the Ga fixed-point-based temperature reference system}

To evaluate the performance of the gallium fixedpoint cell, a thorough estimation of its uncertainty, presented in Table 1, was first performed.

Table 1: The estimated uncertainty of the gallium fixedpoint cavity temperature measurement setup.

\begin{tabular}{|l|l|l|}
\hline Uncertainty Components & $\begin{array}{l}\text { Uncertainty } \\
(\boldsymbol{k}=\mathbf{2}), \mathbf{m K}\end{array}$ & Type \\
\hline Chemical impurities & $0.01^{1}$ & $\mathrm{~B}$ \\
\hline $\begin{array}{l}\text { Atmospheric } \\
\text { pressure variations }\end{array}$ & $0.06^{2}$ & $\mathrm{~B}$ \\
\hline $\begin{array}{l}\text { Hydrostatic } \\
\text { pressure correction }\end{array}$ & $0.01^{3}$ & $\mathrm{~B}$ \\
\hline $\begin{array}{l}\text { Temperature over-hearing } \\
\text { to the block }\end{array}$ & $0.01^{4}$ & $\mathrm{~A}$ \\
\hline Repeatability & $0.22^{5}$ & $\mathrm{~A}$ \\
\hline Nanovoltmeter stability & $1.2^{6}$ & $\mathrm{~B}$ \\
\hline Total: & $\mathbf{1 . 2}$ & \\
\hline
\end{tabular}

The uncertainties due to chemical impurities, atmospheric pressure, and the hydrostatic pressure correction, were all, according to footnotes 1-3, estimated to be below $0.1 \mathrm{mK}$.

To estimate the uncertainty due to temperature over-hearing, a series of melting curves were recorded while the system was stressed, i.e. the temperature of the block, the cavity, and the breadboard were deliberately changed in order to introduce overhearing effects. The only substantial overhearing was found between the block and the Ga fixed-point cell. It was found that a change of $1 \mathrm{~K}$ of the temperature of the block influenced the thermocouple reading 1 $\mathrm{mK}$. Assuming an uncertainty in the assessment of the temperature of the block of $10 \mathrm{mK}$ this will therefore solely give an uncertainty in the assessed temperature of $10 \mu \mathrm{K}$.

To estimate the stability of the system, the temperature of the cavity measured by the thermocouple, $T_{T C}$, was first compared with that measured by the Pt-100 probes, $T_{c a v}$. The difference between the two is shown in Fig. 5 for a single heating cycle.

\footnotetext{
${ }^{1}$ Gallium 7N sourced from Aldrich lot MKCH6524.

${ }^{2}$ Estimated from: variation of $10 \mathrm{kPa}$ over a year [8] and ( $d T / d P$ ) of $-2.0 \times 10^{-8} \mathrm{~K} / \mathrm{Pa}[9]$.

${ }^{3}$ Estimated from: mounting point of probe and $(d T / d l)$ of $-1.2 \times 10^{3} \mathrm{~K} / \mathrm{m}$ [9].
}

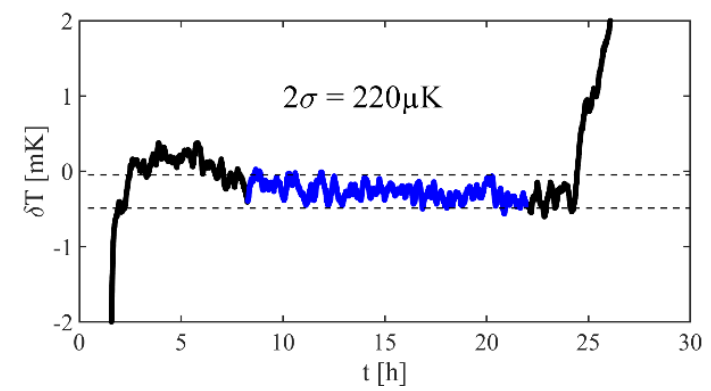

Figure 5: The difference in cavity temperature measured with the thermocouple, $T_{T C}$, and the Pt-100 probes, $T_{c a v}$. The blue markers corresponds to the data evaluated. The dashed lines correspond to $\pm 2 \sigma$ i.e. $\pm 220 \mu \mathrm{K}$.

The stability of this assessment is given by a combination of the stability of the actual temperature of the gallium in the fixed-point cell, that of the thermocouple measurement, and that of the cavity temperature measured with Pt-100 probes. To evaluate the influence of the latter, the stability of the resistance measurement module was monitored by the use of a standard resistor. Figure 5 shows the Ga melting cycle out of five that showed the highest resistance measurement stability.

The part of the melting cycle during which temperature was assessed was taken as half of the melting plateau (the blue markers in Fig. 5). Its stability was estimated (as two standard deviations over the $13 \mathrm{~h}$ period) to $220 \mu \mathrm{K}$. Since the assessment of the stability of the measurement module was found to represent $80 \mu \mathrm{K}$, the combined stability of the fixedpoint cell and thermocouple measurement can be assumed to be somewhat smaller than $220 \mu \mathrm{K}$. This result is in agreement with the expected melting curve of $7 \mathrm{~N} \mathrm{Ga}[10]$.

It was found that the main contribution to the uncertainty came from the nanovoltmeter, which provided an uncertainty of $1.2 \mathrm{mK}$. Since all other contributions to the uncertainty solely were fractions of this value, it also constitutes the total estimated uncertainty of the $\mathrm{Ga}$ fixed-point cell based temperature measurement setup. This corresponds to a relative uncertainty of the assessment of temperature of $4 \mathrm{ppm}$.

\subsection{A comparison between the two means to assess cavity temperature; the Pt-100 sensors and the thermocouple system}

To demonstrate the performance of the temperature regulation and assessment system, a series of measurements was performed at a pressure of 11620 $\mathrm{Pa}$ (produced by a dead weight pressure balance) over

\footnotetext{
${ }^{4}$ Assuming an error of $10 \mathrm{mK}$ in the assessed block temperature. Correction formula $0.001 \times\left(T_{B l o c k}-T_{G a}\right)$.

${ }^{5}$ Measurement $2 \times$ STD over $13 \mathrm{~h}$.

${ }^{6}$ Estimated from: Accuracy: $\pm(40 \mathrm{ppm}$ of reading $+4 \mathrm{ppm}$ of range) (90 days).
} 
a single melting cycle $(\sim 90 \mathrm{~h})$. A measurement of various temperatures in the system is shown in Fig. 6 . The red, blue, and black curves represent the measured temperatures of the cavity measured by the Pt-100 sensors, $T_{c a v}$, the thermocouple, $T_{T C}$, and the temperature of the fixed-point cell, $T_{\text {cell }}$, all compared to the nominal melting point of $\mathrm{Ga}, T_{G a}$, respectively.

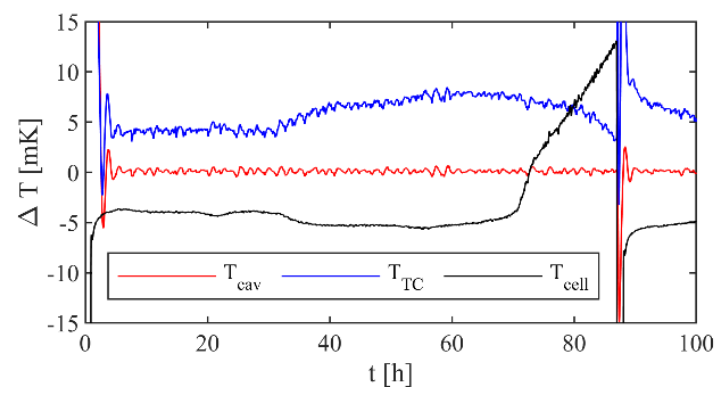

Figure 6: Red curve: the temperature of the cavity assessed by the Pt-100 sensor referenced to the nominal melting point of Ga, i.e. $T_{c a v}-T_{G a}$; Blue curve, the temperature of the cavity assessed by the thermocouple, referenced to the same entity, i.e. $T_{T C}-T_{G a}$; Black curve: the temperature of the Ga fixed-point cell assessed by the Pt-100 sensor referenced to $T_{G a}$, i.e. $T_{\text {cell }}-T_{G a}$.

While the black curve solely is used for monitoring the Ga melting-cycle, the red is used to give feedback for the stabilization of the cavity temperature. Drifts in the temperature assessment by the Pt-100 sensors will therefore be transferred into the temperature of the cavity (the lack of drifts in the red curve indicates that the temperature regulation system works well). The blue curve represents the cavity temperature assessed by the thermocouple. As the uncertainty in this assessment (given by Table 1) is smaller than its drift, the assessed $T_{T C}$ represents the actual temperature of the cavity.

\subsection{Assessment of the Ga fixed-point referenced Invar-based DFPC system}

To characterize the combined Ga fixed-point cell and thermocouple system regarding its ability to assess pressure, a comparison between the pressures assessed by the refractometer using the two different means of temperature assessment was performed. The data taken during the melting cycle presented above were converted to refractivity by the use of Eq. (1).

In one of the cases, the pressure was assessed from this data by use of the temperature measured by the Pt-100 sensors (the red curve in Fig. 6) placed in the cavity spacer, providing $T_{c a v}$. In the other case, the pressure was assessed (from the same refractivity data) by use of the temperature assessed by the combined $\mathrm{Ga}$ fixed-point cell and thermocouple system, i.e. $T_{T C}$ (the blue curve in Fig. 6).

The refractometry system and the piston gauge was exposed to 3600 gas filling-and-empting cycles according to the normal procedures of the GAMOR methodology $[3,4,6]$. Each cycle lasted $100 \mathrm{~s}$ while the entire measurement campaign took 100 hours.

The difference in pressure assessed by the refractometer and that set by the pressure balance, evaluated by the two different assessed temperatures, are presented in Fig 7 (assessed as $T_{c a v}$, the lower black-and-red curve, and as $T_{T C}$, the upper blackand-blue curve).



Figure 7: The pressure difference between the set pressure of the pressure balance and the pressures assessed by the refractometry evaluated using temperatures assessed by use of Pt-100 probes (black-and-red curve), $T_{c a v}$, and the thermocouple referenced to the Ga cell (black-and-blue curve), $T_{T C}$. The dashed lines correspond to $\pm 2 \sigma$, which is $\pm 120 \mathrm{mPa}$ for the Pt-100 based measurements and \pm 32 $\mathrm{mPa}$ for the thermocouple-based assessments.

The coloured parts of the curves represent the centre half of the Ga melting-plateau. Evaluation of the system was made during this time period. The lowermost curve, representing assessment of the pressure difference by the use of the Pt-100 sensors, was found to have a $2 \sigma$-deviation over $40 \mathrm{~h}$ of 120 $\mathrm{mPa}$, while the same measurement data, evaluated by use of the thermocouple system, was $32 \mathrm{mPa}$. For the pressure used $(11620 \mathrm{~Pa})$, this corresponds to stabilities of 10.4 and $2.8 \mathrm{ppm}$, respectively. This confirms the statement above that the temperature assessed by the combined Ga fixed-point cell and thermocouple system provides the actual temperature of the cavity.

\section{SUMMARY}

In summary, this work presents a DFPC-based refractometer made of Invar with a temperature regulation and assessment system that is based on a regulation of the temperature of the cavity based on the cavity temperature assessed by use of three PT100 sensors, while the temperature of the cavity is assessed by the use of a thermocouple referenced to a Ga fixed-point cell. It was first noted that an Invarbased refractometer can provide a precision below $0.1 \mathrm{ppm}$ for time scales between 400 and $1100 \mathrm{~s}$. The temperature regulation and assessment system was then described and scrutinized in some detail. An estimation of the uncertainties in the temperature measurement system indicated that it provides a total 
uncertainty of $1.2 \mathrm{mK}$ ( $4 \mathrm{ppm})$, dominated by the stability of the nanovoltmeter used for assessment of the thermocouple voltage.

This work has shown that the combination of Invar spacer and Ga fixed-point cell can provide a basis for a self-contained system that only needs a pure gas supply and accurate frequency references to realize the Pascal. This is an important step towards the dissemination of the Pascal through fundamental principles.

This project (QuantumPascal, 18SIB04) has received funding from the EMPIR programme cofinanced by the Participating States and from the European Union's Horizon 2020 research and innovation programme. The authors also acknowledge support from the Swedish Research Council (VR) (Project No. 621-2015-04374); the Umeå University Industrial Doctoral School (IDS); the Vinnova Metrology Programme (Project Nos. 2018-04570 and 2019-05029); and the Kempe Foundations (Project No. 1823, U12).

\section{REFERENCES}

[1] P. F. Egan, J. A. Stone, J. H. Hendricks, J. E. Ricker, G. E. Scace, G. F. Strouse, "Performance of a dual Fabry-Perot cavity refractometer," Opt. Lett. vol. 40, 3945-3948, 2015.

[2] P. F. Egan, J. A. Stone, J. E. Ricker, J. H. Hendricks, "Comparison measurements of low-pressure between a laser refractometer ultrasonic manometer," Rev. Sci. Instrum., vol. 87, 053113, 2016.
[3] I. Silander, T. Hausmaninger, M. Zelan, O. Axner, "Gas modulation refractometry for high-precision assessment of pressure under non-temperaturestabilized conditions", J. Vac. Sci. \& Technol. A, vol. 36, 03E105, 2018.

[4] I. Silander, T. Hausmaninger, C. Forssén, M. Zelan, O. Axner, "Gas equilibration gas modulation refractometry for assessment of pressure with subppm precision", J. Vac. Sci. \& Technol. B, vol. 37, 042901, 2019.

[5] O. Axner, I. Silander, C. Forssén, J. Zakrisson, M. Zelan, "Ability of gas modulation to reduce the pickup of fluctuations in refractometry," JOSA B, vol. 37, 1956-1965, 2020.

[6] I. Silander, C. Forssén, J. Zakrisson, M. Zelan, O. Axner, "Invar-based refractometer for pressure assessments," Opt. Lett., vol. 45, 2652-2655, 2020.

[7] Topham, T., Bingham, G., Latvakoski, H. et al. Observational study: microgravity testing of a phasechange reference on the International Space Station. npj Microgravity, vol. 1, 15009, 2015.

[8] Retrieved from (in Swedish) www.smhi.se/kunskapsbanken/meteorologi/lufttryc $\mathrm{k}-1.657$.

[9] "Guide to the Realization of the ITS-90", www.bipm.org/en/committees/cc/cct/guideits90.html.

[10] Nakano, T., Tamura, O. \& Sakurai, H. Realization of the Gallium Triple Point at NMIJ/AIST. Int J Thermophys, vol. 29, 112-118, 2008.

[11] J. Zakrisson, I. Silander, C. Forssén, M. Zelan, O. Axner, "Procedure for robust assessment of cavity deformation in Fabry-Pérot based refractometers" J. Vac. Sci. Technol. B, vol. 38, 054202, 2020. 